\title{
Humoral response to SARS-CoV-2 vaccines in people living with HIV
}

\author{
Sebastian Noe ${ }^{1}$ (1) Nino Ochana ${ }^{1} \cdot$ Carmen Wiese $^{1} \cdot$ Farhad Schabaz $^{1} \cdot$ Ariane Von Krosigk $^{1} \cdot$ Silke Heldwein $^{1}$. \\ Rudolf Rasshofer $^{1} \cdot$ Eva Wolf $^{2}$. Celia Jonsson-Oldenbuettel ${ }^{1}$
}

Received: 7 September 2021 / Accepted: 12 October 2021 / Published online: 25 October 2021

(c) The Author(s), under exclusive licence to Springer-Verlag GmbH Germany 2021

\begin{abstract}
Purpose To describe the humoral immune response to COVID-19 vaccines in people living with HIV and identify factors associated with the magnitude of anti-SARS-CoV-2 antibody concentrations.

Methods Retrospective analysis of electronic patient files in a big single HIV center in Munich, Germany. Non-parametric methods were used for descriptive and comparative statistics. Generalized linear models were used to analyze associations of general and HIV-specific variables with anti-SARS-CoV-2 antibody concentrations after standard vaccination.

Result Overall, 665 people living with HIV were included into the analysis (median age: 53 [IQR: 43; 59]), 560 [84.2\%] males). Median concentration of anti-SARS-CoV-2-antibodies was 1400 (IQR 664; 2130) BAU/mL. In 18 (2.7\%) subjects, antibody concentrations below the threshold of $34 \mathrm{BAU} / \mathrm{mL}$ were found. Among PLWH with CD4 cell count $<200$ cells/ $\mu \mathrm{L}$, median anti-SARS-CoV-2-Abs were 197 (IQR 44.6; 537.2) as compared to 1420 (IQR 687; 2216) for the group $\geq 200$ cells/ $\mu \mathrm{L}(p<0.001)$. In a cumulative logit model, a vaccination scheme including an mRNA vaccine [OR: 4.64 (3.58; 6.02)], being female [OR: $2.14(1.76 ; 2.61)$ ], and having higher CD4 cells [OR per 100 cells/ $\mu \mathrm{L}: 1.06(1.04 ; 1.08)$ ] were significantly associated with anti-SARS-CoV-2 antibody concentrations in higher quartiles, when adjusted for the time after vaccination. Conclusion We found a robust antibody response in people living with HIV undergoing standard vaccination against SARSCoV-2. mRNA-containing vaccination schemes, being female, and having a higher CD4 cell count was associated with a higher concentration of antibodies in people living with HIV in our study sample. Particularly in the subgroup with CD4 cell counts $<200$ cells $/ \mu \mathrm{L}$, antibody response was poor.
\end{abstract}

Keywords COVID-19 · COVID-19 vaccines $\cdot$ SARS-CoV-2 $\cdot$ HIV $\cdot$ CD4

\section{Introduction}

The global spread of the severe acute respiratory syndrome corona virus 2 (SARS-CoV-2) starting in late 2019 has turned into a pandemic that has been causing more than $230,000,000$ infections and 4,800,000 deaths worldwide as of October 2021 [1]. The disease is still not only challenging health care systems all over the world, but also causing massive disturbance in everyday life of many people and immense financial consequences. Preventing the spread of SARS-CoV-2 infection has therefore become a major goal in the management of the pandemic. While some risk factors

Sebastian Noe

sno@mvz-mag.de

1 MVZ München Am Goetheplatz, Waltherstr. 32, 80337 Munich, Germany

2 MUC Research, Munich, Germany for a higher risk of acquiring SARS-CoV-2 infection or suffering from a more severe course have been determined, including age and obesity amongst others, the role of HIV infection needs a differentiated consideration: while people living with HIV (PLWH) might not be at a particular risk in general, there is increasing evidence for a more severe course of COVID-19 in PLWH with low CD4 cells and/or a low CD4 cell nadir [2-8]. As a consequence, PLWH were prioritized to receiving anti-SARS-CoV-2 vaccines in many countries. However, while data on the effects of SARSCoV-2 vaccines in PLWH is increasing, it is still sparse [9, 10]. It has been demonstrated before that (for some vaccines) the degree of protection and/or the humoral response in PLWH (children and adults) differs from the one observed in a general population $[11,12]$. It was debated, that the degree of protection against SARS-CoV-2 infection might be difficult to quantify as it might be comprised not only of the humoral, but also of the cellular response. However, 
recent data seem to imply that the concentration of neutralizing anti-SARS-CoV-2-antibodies (SARS-CoV-2-Abs) is highly predictive for the overall protection against SARSCoV-2 infection [13]. Also, in people with 'breakthrough' infections after standard immunization, lower concentrations of neutralizing antibodies around the time of infection were found when compared to controls and higher antibody concentrations were in turn associated with lower infectivity [14]. The amount of (neutralizing) antibodies developed after standard vaccination with COVID-19 vaccines might therefore be a good surrogate marker of protection from SARS-CoV-2 infection and/or a severe course of COVID-19.

This study aimed to describe the development of SARSCoV-2-Abs in PLWH after standard vaccination in a single HIV center in Munich, Germany, and to investigate the association of general and HIV-specific factors with the development of SARS-CoV-2-Abs.

\section{Methods}

This non-interventional, retrospective study was performed using electronic patient files from a single HIV research and clinical care center in Munich, Germany (MVZ München am Goetheplatz), that routinely offered measurement of antiSARS-CoV-2-Abs within clinical routine. Due to the study design, the study did not require ethics approval. Among all subjects with available measurements of SARS-CoV-2-Abs between the last dose of the standard immunization and any further dose of SARS-CoV-2 vaccine between April 1 and August 31 2021, people without documented HIV-1 infection, with a documented history of SARS-CoV-2 infection, or with missing information on date and scheme of vaccination were excluded. For each subject the mode of standard vaccination was recorded as consisting of either two doses of a mRNA vaccine (Comirnaty or COVID-19 Vaccine Moderna), two doses of the COVID-19 Vaccine AstraZeneca, or one dose of the COVID-19 Vaccine Janssen, or one dose of a mRNA vaccine after a first dose of the COVID-19 Vaccine AstraZeneca (heterologous vaccination). For the quantitative determination of anti-trimeric spike protein specific IgG antibodies, the LIAISON SARS-CoV-2 TrimericS IgG Assay (Diasorin, Italy) was used; results are presented in binding antibody units per $\mathrm{mL}(\mathrm{BAU} / \mathrm{mL})$. CD4 cell count and HIV-1 RNA-levels were documented from the same day as the determination of SARS-CoV-2-Abs or, where not available from the same day, adopted from the next closest (earlier) laboratory evaluation within a range of a maximum of 3 months. Other variables of interest that were recorded for the purpose of this study were sex, mode of HIV infection, ethnicity, date of HIV diagnosis, CD4 nadir (lowest CD4 cell count before suppressive antiretroviral therapy or during a pause of antiretroviral therapy), current antitretroviral therapy regimen at the time of the blood sampling for laboratory tests, and history of AIDS-defining diseases. Frequencies and percentages or proportions and medians with interquartile ranges (IQR) or means and standard deviations (SD) were used for descriptive statistics. Comparison of antibody concentrations between the different schemes of standard vaccination was performed using the Jonckheere-Terpstra test, assuming an ordered response among the groups (homologues mRNA vaccination $>$ heterologous vaccination $>$ homologous vaccination COVID19 Vaccine AstraZeneca > singular vaccination COVID-19 Vaccine Janssen). For other continuous variables, KruskalWallis test was used, while $\chi^{2}$-test was used for the comparison of categorical variables. Quartiles of anti-SARSCoV-2-antibody concentrations observed in this study were calculated and each subject was assigned to any of the four quartiles according to their antibody concentrations. Association with Anti-SARS-CoV-2-Abs was explored visually using a scatter plot and correlation. This ordinal categorical variable was used as the dependent variable in a generalized linear model using a cumulative logit link under the assumption of common slopes. Age, sex, basic immunization scheme (mRNA-containing yes/no), time between the last dose of basic immunization and assessment of antibodies, HIV-1 RNA-levels (< 50 copies/mL yes/no), current CD4 cell count $[$ cells $/ \mu \mathrm{L}]$, and history of AIDS-defining diseases [yes/no] were used as independent variables. CD4 nadir was not included into the model due to a high number of missing data. Univariate, explorative analysis for the association of CD4 cell nadir and anti-SARS-CoV-2-Abs was performed using Spearman's correlation. After univariate analysis, a multiple regression model was fit using a forward selection approach, adding all variables that significantly contributed to the model fit, one at a time, using likelihood ratio tests to compare the models. The final model was compared to the same model under the assumption of non-equal slopes. Data analysis was performed using $\mathrm{R}$ (Version 4.0.3). Unless declared otherwise, $p<0.05$ were considered to be statistically significant.

\section{Results}

From 1055 subjects with anti-SARS-CoV-2-Abs available from electronic patient files, 665 (63\%) were included into the final analysis after applying in- and exclusion criteria (Appendix A.1); baseline characteristics can be found in Table 1. Standard immunization was performed with two doses of a mRNA vaccine in $590(88.7 \%)$, two doses of the COVID-19 Vaccine AstraZeneca in $31(4.7 \%)$, or one dose of the COVID-19 Vaccine Janssen in 15 (2.3\%). For the subgroup of PLWH receiving a homologous mRNA vaccination scheme, $582(98.6 \%)$ and $8(1.4 \%)$, received the 
Table 1 Baseline characteristics of all participants in this study

\begin{tabular}{lll}
\hline Age [years], median (IQR) & 53 & $(43 ; 59)$ \\
Missing data, $n(\%)$ & 0 & $(0)$ \\
Male, $n(\%)$ & 560 & $(84.2)$ \\
Missing data, $n(\%)$ & 0 & $(0)$ \\
Caucasian, $n(\%)$ & 515 & $(85.4)$ \\
Missing data, $n(\%)$ & 62 & $(9.3)$ \\
HIV-PCR $<50$ copies/mL, $n(\%)$ & 622 & $(93.5)$ \\
Missing data, $n(\%)$ & 0 & $(0)$ \\
CD4 cell [cells/ $\mu \mathrm{L}]$, median $(\mathrm{IQR})$ & 708 & $(524 ; 912)$ \\
Missing data, $n(\%)$ & 0 & $(0)$ \\
CD4 cell nadir [cells/ $\mu \mathrm{L}$ ], median (IQR) & 264 & $(143.5 ; 388.2)$ \\
Missing data, $n(\%)$ & 269 & $(40.5)$ \\
History of AIDS-defining disease, $n(\%)$ & 80 & $(12.4)$ \\
Missing data, $n(\%)$ & 21 & $(3.2)$ \\
\hline
\end{tabular}

BioNTech and the Moderna mRNA vaccine, respectively. Due to the massive difference in the groups size, the two vaccines were not compared to one another. In 29 (4.4\%) the standard immunization was completed using a sequence of a mRNA vaccine after a first dose of the COVID-19 Vaccine AstraZeneca. Anti-SARS-CoV-2-Abs concentrations for each of the standard immunization groups can be found in Table 2: while the main characteristics of the four groups were comparable, significantly higher concentrations of anti-SARS-CoV-2-Abs were found in PLWH undergoing an mRNA-containing standard vaccination scheme (homo- or heterologous) when compared to schemes only consisting of vector-based vaccines. However, also the time interval between standard vaccination and measurement of antiSARS-CoV-2-Abs differed between the groups. Median time between last vaccination and measurement of anti-SARSCoV-2-Abs was 49 (IQR 35; 63) days. Median concentration of SARS-CoV- 2-Abs was 1400 (IQR 664; 2130) BAU/ $\mathrm{mL}$. In 18 (2.7\%) PLWH, antibody concentrations below the threshold of $34 \mathrm{BAU} / \mathrm{mL}$ were found.

The association of the time interval between the last shot of the basic immunization and the measurement of antiSARS-CoV-2-Abs for the first 12 weeks is illustrated in Fig. 2: an increase of the mean concentration of antibody concentrations was found from week one with a maximum at week three and a decrease thereafter. Between weeks 8 and 12 , mean antibody concentrations were comparable. Of note, there were 14 (2.1\%) PLWH with a current CD4 cell concentration $<200$ cells $/ \mathrm{mL}$. Among PLWH with CD4 cell count $<200$ cells/ $\mu \mathrm{L}$, median anti-SARS-CoV-2-Abs was 197 (IQR 44.6; 537.2) as compared to 1420 (IQR 687; 2216) $(\mathrm{p}<0.001)$. A scatter plot of the CD4 cell nadir and the concentration of SARS-CoV-2-Abs after a standard vaccination is displayed in Fig. 1, including a median regression line for antibody concentrations by CD4 cell nadir. Correlation

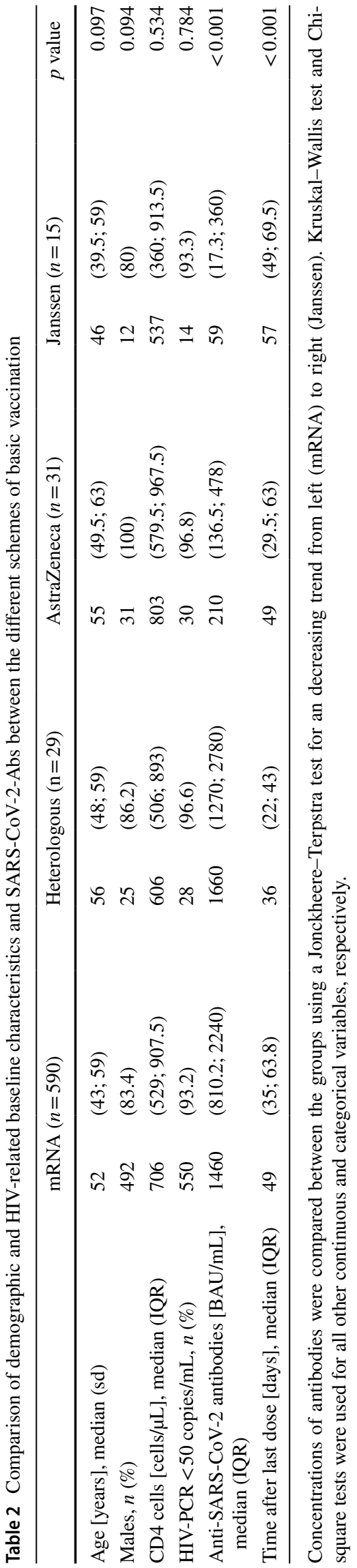


Fig. 1 Scatter-plot on the association between CD4 cell nadir and concentrations of antiSARS-CoV-2-antibodies after standard vaccination. The solid line indicates a median regression line for antibody- concentrations by $\mathrm{CD} 4$ cell nadir

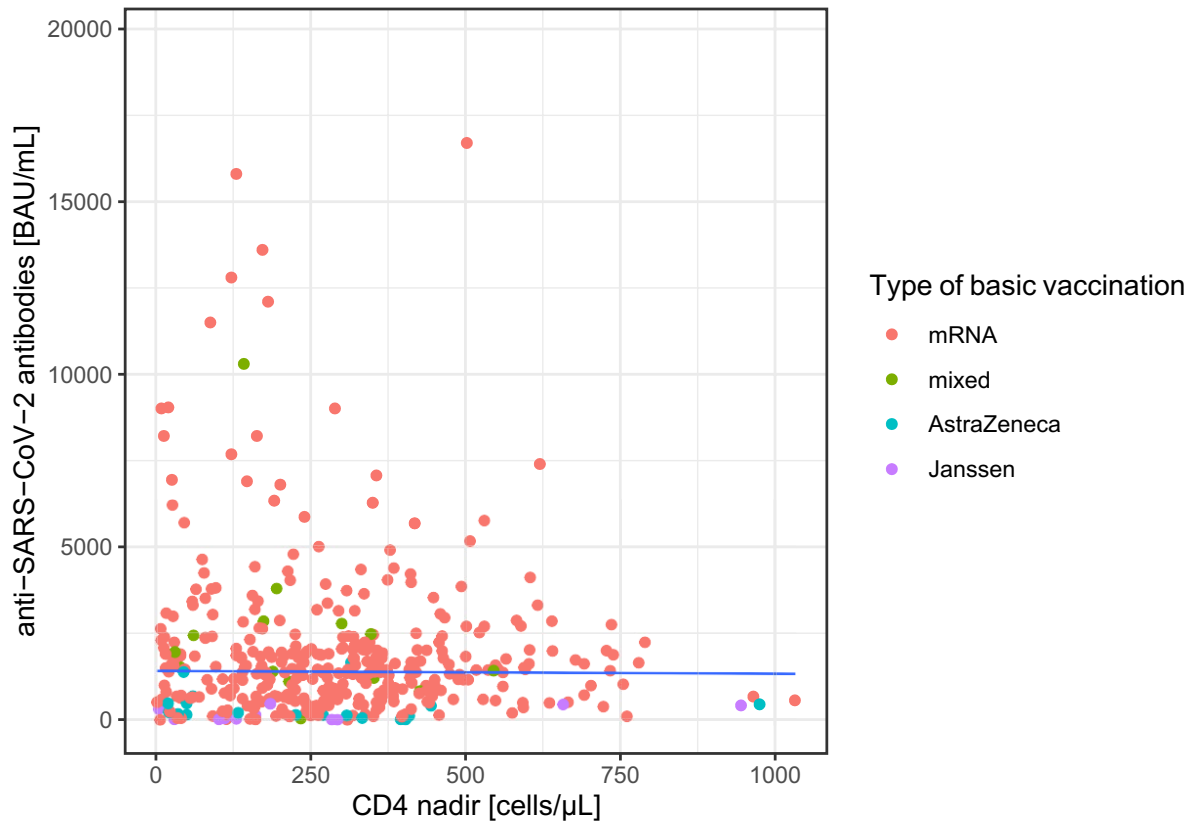

between concentrations of SARS-CoV-2-Abs with CD4 cell nadir was $\rho=-0.01(p=0.912)$. The results of simple and multiple regression using a cumulative logit model are displayed in Table 3: sex, time after completion of the standard vaccination, use of an mRNA-containing vaccination scheme, and current CD4 cells demonstrated significant associations with the concentration of anti-SARS-CoV-2 in muliple regression analysis.

\section{Discussion}

Our data demonstrate an on average solid humoral response following standard vaccination against SARS- CoV-2 infection in PLWH, comparable to the concentrations of antibodies found in a sample of people from a 'general' population after COVID-19 with the same assay [15]; only in 18 PLWH the antibody concentrations were found to be lower than the assay specific threshold indicating 'positivity' for antiSARS-CoV-2-Abs. Although it has to be kept in mind that our data are not longitudinal, a maximum in the humoral response seems to be found around week 3 after the last dose of the standard vaccination scheme (Fig. 2). Of interest

Table 3 Results for simple and multiple ordinal regression on the quartiles of antibodiy concentrations

\begin{tabular}{|c|c|c|c|c|c|c|}
\hline & \multicolumn{3}{|c|}{ Simple } & \multicolumn{3}{|c|}{ Multiple } \\
\hline & OR & $95 \% \mathrm{CI}$ & $p$ value & OR & $95 \% \mathrm{CI}$ & $p$ value \\
\hline Age [years] & 0.99 & $(0.98 ; 1.01)$ & 0.385 & & & \\
\hline Sex [being female] & 2.12 & $(1.46 ; 3.09)$ & $<0.001$ & 2.14 & $(1.76 ; 2.61)$ & $<0.001$ \\
\hline Time after basic vaccination [weeks] & 0.82 & $(0.78 ; 0.86)$ & $<0.001$ & 0.80 & $(0.78 ; 0.82)$ & $<0.001$ \\
\hline Assessment $\leq 3$ weeks after basic vaccination [yes] & 2.49 & $(1.46 ; 4.27)$ & $<0.001$ & 0.05 & $(0.01 ; 0.14)$ & 0.007 \\
\hline mRNA-containing scheme [yes] & 3.99 & $(2.46 ; 6.47)$ & $<0.001$ & 4.64 & $(3.58 ; 6.02)$ & $<0.001$ \\
\hline HIV-1 RNA level $<50$ copies/mL [yes] & 1.82 & $(1.05 ; 3.16)$ & 0.033 & & & \\
\hline CD4 cell count [per $100 \cdot$ cells $/ \mu \mathrm{L}]$ & 1.05 & $(1.01 ; 1.10)$ & 0.016 & 1.06 & $(1.04 ; 1.08)$ & 0.006 \\
\hline History of AIDS [yes] & 0.96 & $(0.62 ; 1.47)$ & 0.840 & & & \\
\hline
\end{tabular}

The cumulative logit model was fitted under the assumption of common slopes. For the multiple regression model, the overall time effect consists of three components, being the time interval between the last shot of the basic immunization cycle [weeks], laboratory assessment being before the expected maximum humoral response in week three, and the interaction between the two $(\beta=1.2, p=0.004)$. Therefore, while the odds of belonging to higher quartiles or antibody concentrations increases over the first 3 weeks, it decreases afterwards. 
Fig. 2 Mean anti-SARS-CoV2-Abs concentration by time within the first 12 weeks after the final vaccination of the standard vaccination. A 4th degree polynomial smoothing function was fit to the change of mean concentrations over time

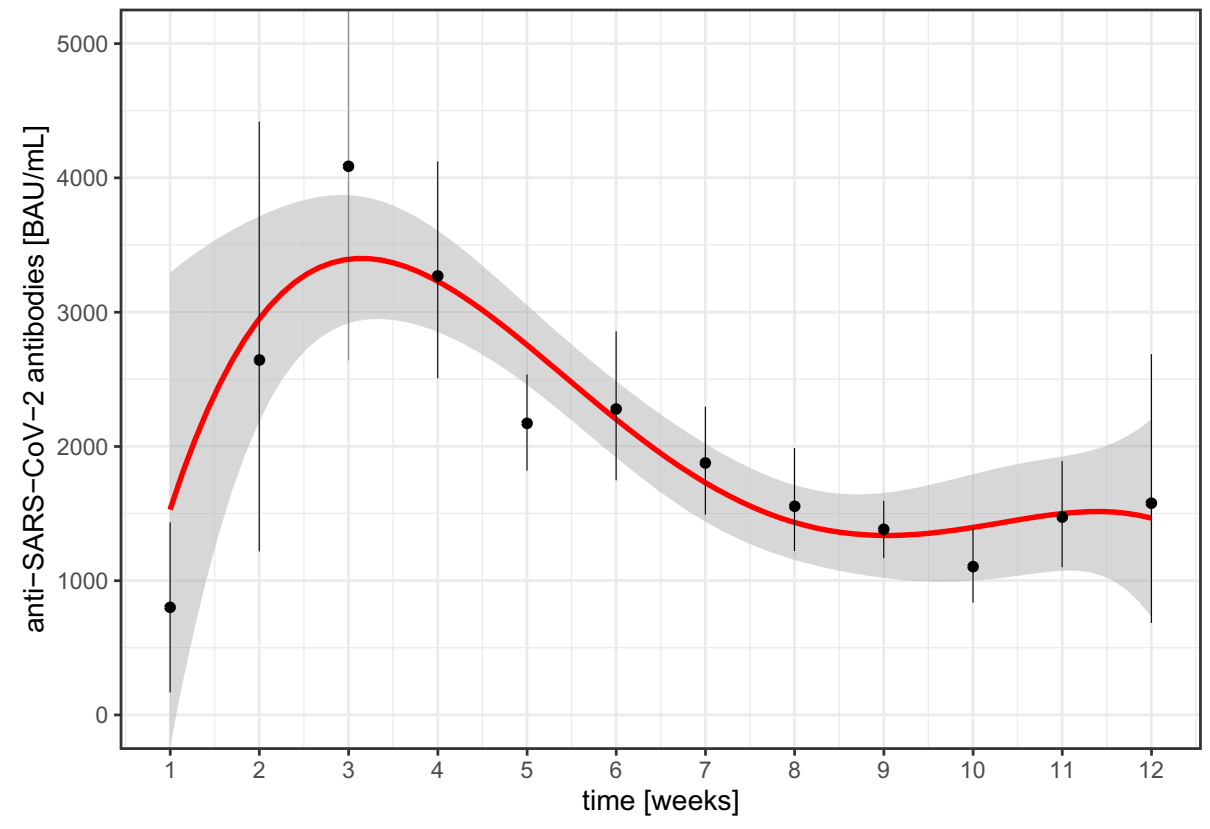

are the marked differences observed with different schemes of standard immunization. While mRNA-containing vaccination schemes (either homologous or heterologous) demonstrated the highest response, the vector-only vaccination schemes were associated with much lower median antibody concentrations (Table 2). Also, the observed humoral response following homologous and heterologous vaccination with a mRNA vaccine were comparable. Even though the heterologous vaccination groups was much smaller than the mRNA-only group and demonstrated a wider spread of the data, these findings are in line with previous findings from non-HIV study samples [16]. In our data, being female was significantly associated with a higher probability of demonstrating antibody concentrations in higher rather than in lower quartiles. This seems to be in line with data from 'general' populations, where the humoral response following SARS-CoV-2 vaccinations was also found to be more pronounced in women, when compared to men [17]. Among HIV-specific factors, only the current CD4 cell concentration was significantly associated with antibody response. In particular, there was a markedly lower response in PLWH with a CD4 cell count $<200$ cells $/ \mu \mathrm{L}$. The effect throughout the entire range of CD4 cells (about $6 \%$ increase in the odds of belonging to a higher quartile per 100 additional cells, keeping all other conditions fixed) was rather low. However, it must be kept in mind, that cellular immune response plays an important role in SARS-CoV-2 [18]. This component was not taken into account by our study and could also differ between vector- and mRNA-based vaccination regimens. While in general the antibody concentration following vaccination seems to allow a good prediction of an overall degree of protection from infection with SARS-CoV-2 and a severe course of COVID-19, it could be assumed that the degree of humoral response is paralleled by the cellular immune response in most people. Therefore, anti-SARS-CoV-2-Abs might be understood as a surrogate for an overall immune response. However, it has been demonstrated, that in some populations the cellular immune response seems to 'stay behind' the humoral one [19]. This might also be true in PLWH relevantly depleted of CD4 cells so that this subset of PLWH might be affected not only by a higher risk for a low humoral but also a low cellular immune response. More information on the cellular immune response following SARS-CoV-2 vaccination in PLWH and conditional on their CD4 cell concentration would be highly appreciated.

Our study has several limitations. In some of the subjects included into this analysis, subclinical SARS-CoV-2 infection might have occurred prior to vaccination or the knowledge about SARS-CoV-2 infection was missing. This might have been of substantial impact on the concentration of SARS-CoV-2-Abs measured in these individuals. Furthermore, we do not have information on the intake of antiinflammatory drugs after the vaccinations that might be of relevance when it comes to humoral immune response [20]. Furthermore, results from our study resulted from random sampling rather than a randomized and therefore representative sample of the cohort under investigation. This might particularly have led to an underrepresentation of elderly PLWH that might be particularly prone to poor humoral response to vaccines. Finally, while commercially available assays to detect antibodies, including neutralizing antibodies, against SARS-CoV-2 have been used in many studies including ours, the 'gold-standard' for the determination of the neutralizing capabilities of antibodies are (pseudo-) 
virus-based assays, that are not used in clinical routine and therefore were not available for analysis in our study. In this context it is important to acknowledge that due to a lower specificity of the high-throughput methods like used in our study when compared to the 'gold-standard' [21], 'positivity' as defined by the specific threshold of an assay may not be confused with protection [22] and therefore, more information on the meaning of antibody concentrations and their protective capabilities is needed.

Summarizing our findings, receiving a mRNA-containing SARS-CoV-2 vaccination scheme as well as being female is associated with a higher probability of demonstrating a higher humoral immune response in people living with HIV after adjusting for the time between final dose of the standard vaccination and time of measurement of anti-SARSCoV-2-Abs. Current CD4 cell concentration was significantly and positively associated with the concentration of anti-SARS-CoV-2-Abs. PLWH with a CD4 cell count $<200$ cells $/ \mu \mathrm{L}$ seem to exhibit a poor humoral response to standard vaccination against COVID-19 which could imply that this population might need additional doses for standard vaccination and/or early re-vaccination. While in general, a 'booster' is currently recommended for people with immunodeficiency diseases about 6 months after the standard vaccination by institutions like the German Robert Koch-Institute (RKI) or the American Centers for Disease Control and Prevention [23, 24], it seems unlikely that this timeframe is equally adequate for PLWH with poor and good immune status given our surrogate data on immune response. The suggestion to optimize the basic immunization with a third dose of an mRNA vaccine as early as four weeks after the second dose, followed by a booster six months thereafter as recommended by the RKI for people with severe immunodeficiency might therefore be a good options for PLWH with CD4 cells $<200$ cells $/ \mu \mathrm{L}$. A similar approach has also been advocated by the British HIV Association [25]. However, more data is needed to verify whether or not this lower humoral response is associated with worse outcomes in terms of risk of infection and course of COVID-19.

\section{Appendix A}

\section{A.1 Patient selection}

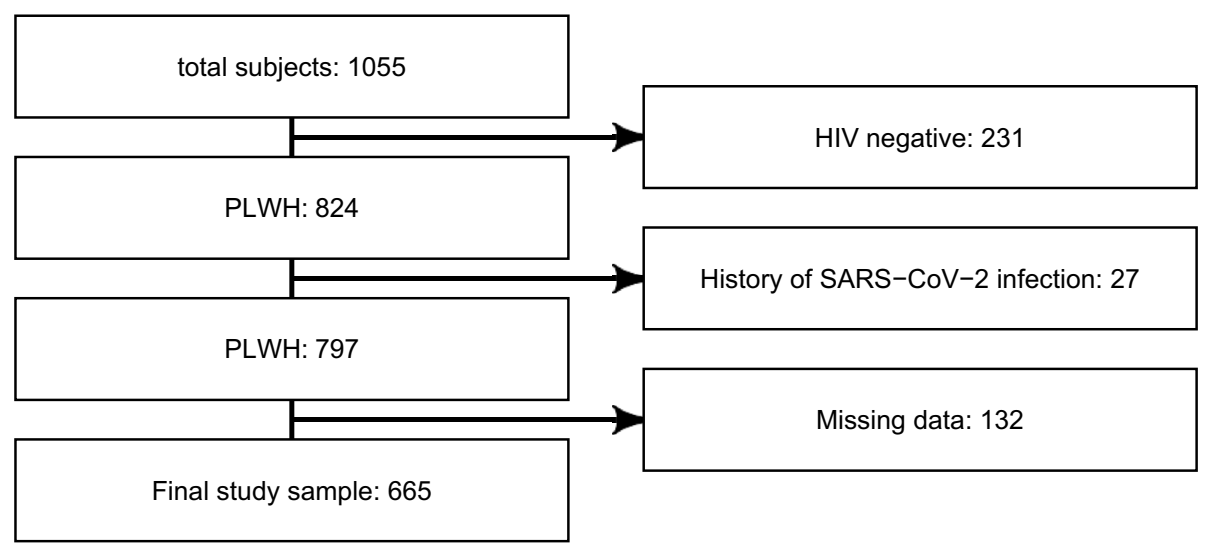

Scheme on the subject selection: out of originally 774 subjects with data on SARS-CoV-2-Abs, 72 subjects with negative HIV-serostatus were excluded from further analysis. Among the remaining 701 subjects, 18 were excluded due to a history of documented SARS-CoV-2 infection. Among the remaining 683 PLWH, 6 had results outside the window of interest (after the last vaccine of the basic vaccination and before any further SARS-CoV-2 vaccination), resulting in a final study sample of 677 .
Acknowledgements We thank the staff of MVZ München am Goetheplatz and the staff of the local laboratory. We thank Dr. Birgit Mück for thoroughly revising the manuscript.

Funding This study did not receive any funding.

Availability of data, material and code Data can be made available by the corresponding author upon reasonable request.

\section{Declarations}

Conflict of interest All authors declare no competing interests. 
Ethics approval This study did not require ethics approval.

\section{References}

1. WHO. https://covid19. who.int/?adgroupsurvey $=$ adgroupsur vey $\}$ \&gclid $=$ CjwKCAjwkvWKBhB4EiwA-GHjFrPCNo5FSrZ2 diQd4YENSf3xYbu8HQWxq6yoYaBjO8E19dDXWs_RLBoC 9FIQAvD_BwE/. Accessed 6 Oct 2021.

2. Tesoriero JM, Swain C-AE, Pierce JL, Zamboni L, Wu M, Holtgrave DR, et al. COVID-19 outcomes among persons living with or without diagnosed HIV infection in new york state. JAMA Netw Open. 2021;4:e2037069.

3. Hoffmann C, Casado JL, Härter G, Vizcarra P, Moreno A, Cattaneo $\mathrm{D}$, et al. Immune deficiency is a risk factor for severe COVID19 in people living with HIV. HIV Med. 2021;22:372-8.

4. Dandachi D, Geiger G, Montgomery MW, Karmen-Tuohy S, Golzy M, Antar AA, et al. Characteristics, comorbidities, and outcomes in a multicenter registry of patients with human immunodeficiency virus and coronavirus disease 2019. Clin Infect Dis. 2021;73(7):e1964-72.

5. Ambrosioni J, Blanco JL, Reyes-Urueña JM, Davies M-A, Sued O, Marcos MA, et al. Overview of SARS-CoV-2 infection in adults living with HIV. Lancet HIV. 2021;8:e294-305.

6. Ho H, Peluso MJ, Margus C, Matias Lopes JP, He C, Gaisa MM, et al. Clinical outcomes and immunologic characteristics of coronavirus disease 2019 in people with human immunodeficiency virus. J Infect Dis. 2021;223:403-8.

7. Chanda D, Minchella PA, Kampamba D, Itoh M, Hines JZ, Fwoloshi S, et al. COVID-19 severity and COVID-19-associated deaths among hospitalized patients with HIV infectionZambia, March-December 2020. Morbidity Mortal Wkly Rep. 2021;70:807.

8. Braunstein SL, Lazar R, Wahnich A, Daskalakis DC, Blackstock OJ. COVID-19 infection among people with HIV in New York city: a population-level analysis of linked surveillance data. Clin Infect Dis. 2021;72(12):e1021-9.

9. Frater J, Ewer KJ, Ogbe A, Pace M, Adele S, Adland E, et al. Safety and immunogenicity of the ChAdOx $1 \mathrm{nCoV}-19$ (AZD1222) vaccine against SARS-CoV-2 in HIV infection: A single-arm substudy of a phase $2 / 3$ clinical trial. Lancet HIV. 2021;8(8):e474-85.

10. Levy I, Wieder-Finesod A, Litchevski V, Biber A, Olmer L, Huppert A, et al. Immunogenicity and safety of the BNT162b2 mRNA COVID-19 vaccine in people living with HIV-1. 2021.

11. Zhan Y, Liu X, Feng Y, Wu S, Jiang Y. Safety and efficacy of human papillomavirus vaccination for people living with HIV: a systematic review and meta-analysis. Int J STD AIDS. 2019;30:1105-15.

12. Adetokunboh OO, Ndwandwe D, Awotiwon A, Uthman OA, Wiysonge CS. Vaccination among HIV-infected, HIV-exposed uninfected and HIV-uninfected children: a systematic review and meta-analysis of evidence related to vaccine efficacy and effectiveness. Hum Vaccines Immunother. 2019;15:2578-89.

13. Khoury DS, Cromer D, Reynaldi A, Schlub TE, Wheatley AK, Juno JA, et al. Neutralizing antibody levels are highly predictive of immune protection from symptomatic SARS-CoV-2 infection. Nat Med. 2021;27(7):1205-11.

14. Bergwerk M, Gonen T, Lustig Y, Amit S, Lipsitch M, Cohen C, et al. COVID-19 breakthrough infections in vaccinated health care workers. N Engl J Med. 2021;385(16):1474-84.

15. Villalta D, Moratto A, Salgarolo V, Da Re M, Giacomello R, Malipiero G, et al. New-generation quantitative immunoassays for SARS-CoV-2 antibody detection: need for harmonization. Ann Lab Med. 2022;42:113-6.

16. Rose R, Neumann F, Grobe O, Lorentz T, Fickenscher H, Krumbholz A. Heterologous immunisation with vector vaccine as prime followed by mRNA vaccine as boost leads to humoral immune response against SARS-CoV-2, which is comparable to that according to a homologous mRNA vaccination scheme. medRxiv. 2021.

17. Demonbreun AR, Sancilio A, Velez ME, Ryan DT, Pesce L, Saber $\mathrm{R}$, et al. COVID-19 mRNA vaccination generates greater IgG levels in women compared to men. J Infect Dis. 2021;224(5):793-7.

18. Hellerstein $\mathrm{M}$. What are the roles of antibodies versus a durable, high quality t-cell response in protective immunity against SARSCoV-2? Vaccine: X. 2020;6.

19. Torres I, Albert E, Gimenez E, Alcaraz MJ, Botija P, Amat P, et al. B and t cell immune responses elicited by the BNT162b2 (pfizer BioNTech) COVID-19 vaccine in nursing home residents. medRxiv. 2021.

20. Chen JS, Alfajaro MM, Chow RD, Wei J, Filler RB, Eisenbarth $\mathrm{SC}$, et al. Nonsteroidal anti-inflammatory drugs dampen the cytokine and antibody response to SARS-CoV-2 infection. J Virol. 2021;95:e0014-21.

21. Muecksch F, Wise H, Templeton K, Batchelor B, Squires M, McCance $\mathrm{K}$, et al. Longitudinal variation in SARS-CoV-2 antibody levels and emergence of viral variants: implications for the ability of serological assays to predict immunity. medRxiv. 2021.

22. Perkmann T, Perkmann-Nagele N, Koller T, Mucher P, Radakovics A, Marculescu R, et al. Anti-spike protein assays to determine SARS-CoV-2 antibody levels: a head-to-head comparison of five quantitative assays. Microbiol Spectr. 2021;9:e00247-e321.

23. https://www.rki.de/DE/Content/Infekt/EpidBull/Archiv/2021/ Ausgaben/39_21.pdf?_blob=publicationFile. Accessed 24 Sep 2021

24. https://www.cdc.gov/coronavirus/2019-ncov/vaccines/boostershot.html. Accessed 30 Sep 2021

25. BHIVA. Recommendations from the joint committee on vaccination and immunisation (JCVI) on COVID vaccine third doses and boosters: Update for service users [Internet]. published online; updated September 24th, 2021. https://www.bhiva.org/recom mendations-from-the-JCVI-on-COVID-vaccine-third-doses-andboosters. 